\title{
Prevalence, social determinants, comorbidities, and patient-reported outcomes of dermographism in Chinese adolescents: a population-based study
}

\author{
Xiaoyu $\mathrm{Gu}^{1}$, Yi Xiao ${ }^{1}$, Juan $\mathrm{Su}^{1}$, Ji $\mathrm{Li}^{1}$, Xiaohui Wang ${ }^{2}$, Bin $\mathrm{Wu}^{3}$, Shijun $\mathrm{Shan}^{4}$, Xiang \\ $\mathrm{Chen}^{5}$, and Minxue Shen ${ }^{5}$ \\ ${ }^{1}$ Xiangya Hospital Central South University \\ ${ }^{2}$ Zhongshan Hospital Xiamen University \\ ${ }^{3}$ The Affiliated People's Hospital of Inner Mongolia Medical University \\ ${ }^{4}$ Xiang'an Hospital, Xiamen University \\ ${ }^{5}$ Xiangya Hospital, Central South University
}

June 10, 2020

To the editor,

Dermographism is a secondary temporal linear cutaneous wheal after scratching, stroking, or rubbing the skin. The prevalence of dermographism was estimated as $4 \%-5 \%$ in general population ${ }^{1}$, with a higher prevalence of $24 \%$ in the pediatric population ${ }^{2}$. From previous reports, dermographism was associated with drug-induced urticaria ${ }^{3}$, hypereosinophilic syndrome ${ }^{4}$, and endocrinopathies. However, the above data were mainly derived from case reports or case-control studies of western countries. Moreover, Bolognia et al proposed that dermographism was not related to the atopy, food allergy, autoimmunity diseases ${ }^{5}$.

We conducted a population-based cross-sectional study in China. The first-year college students in three universities of China who consented to participate underwent health examination and an online questionnaire survey. Diagnosis of skin diseases and inquiry of disease history were performed by dermatologists during the health examination. Dermographism was induced by a scratching test and evaluated by a dermatologist. Annual household income and parents' educational level were determined by a self-reported questionnaire that included six given categories. Itch and pain of the skin were measured by the numeric rating scales. Sleep quality was measured by the Pittsburgh Sleep Quality Index. Symptoms of depression and anxiety were measured by the 2-item Patient Health Questionnaire and Generalized Anxiety Disorder Scale-2, respectively. Mixed logistic models were used to estimate the associations in terms of odds ratios (OR), including crude and adjusted ORs and $95 \%$ uncertainty intervals. The effect sizes were adjusted for the individual-level covariates (sex and socioeconomic indicator) and the random effects of sampling units (university). Statistical analyses were performed with SAS 9.4 (SAS Inc., Cary, USA). $P<0.05$ was considered statistically significant.

A total of 16,167 college students was newly enrolled in three universities located in Changsha, Xiamen, and Hohhot, respectively, in 2018. Among them, 12,127 (response rate 75\%) completed the questionnaire survey and health examination and were finally analyzed. The mean age of the participants was $18.3 \pm 0.8$ years, and $52.7 \%$ were women. The point prevalence of dermographism was $15.6 \%$, with no sex difference. The prevalence of grade 2-3 dermographism was $1.43 \%$. As shown in Table 1, the prevalence of dermographism was positively associated with socioeconomic status indicators (trend $P<0.001$ ). Dermographism was significantly associated with a series of skin comorbidities (Table 2), including atopic dermatitis (adjusted OR=1.39, $P$ 
$=0.008)$, chronic urticaria $(\mathrm{OR}=2.56, P<0.001)$, acne vulgaris $(\mathrm{OR}=1.29, P<0.001)$, and warts $(\mathrm{OR}=2.07$, $P=0.012$ ). In contrast, dermographism was not significantly associated with a self-reported history of asthma or allergic rhinitis or other diagnosed skin disorders. Regarding other patient-reported outcomes (Table $2)$, dermographism was only significantly associated with itch $(\mathrm{OR}=1.20, P=0.005)$.

The high prevalence suggests that dermatologists should not neglect to test relevant patients for dermographism in order to avoid misdiagnosis, especially in patients of younger age and with comorbid conditions such as atopic dermatitis and symptoms of itch. The significant correlation between dermographism and socioeconomic status indicators may be attributable to urbanization, air pollution, and changes in dietary habits. In conclusion, dermographism affects a substantial proportion of Chinese young adults, and is associated with higher socioeconomic status, comorbidities, and symptoms of itch.

\section{References}

1. Jedele KB, Michels VV. Familial dermographism. Am. J. Med. Genet. 1991;39(2):201-203.

2. Martorell A, Sanz J. [Round Table: urticaria with a physical cause]. Allergol Immunopathol (Madr). $1999 ; 27(2): 85-96$.

3. Warner DM, Ramos-Caro FA, Flowers FP. Famotidine (pepcid)-induced symptomatic dermatographism. J. Am. Acad. Dermatol. 1994;31(4):677-678.

4. Cooper MA, Akard LP, Thompson JM. Hypereosinophilic syndrome: long-term remission following allogeneic stem cell transplant in spite of transient eosinophilia post-transplant. Am. J. Hematol. 2005;78(1):33-36.

5. Bolognia JL, Schaffer JV, Cerroni L. Dermatology. 4th ed. Singapore, SG: Elsevier; 2018. 344 p.

Table legends

Table 1. Prevalence of dermographism by sociodemographic characteristics

Table 2. Association of dermographism with comorbidities and patient-reported outcomes

Table 1. Prevalence of dermographism by sociodemographic characteristics

\begin{tabular}{lllll}
\hline & & & Grade, $\%$ & Grade, $\%$ \\
Characteristics & Total, n (\%) & Prevalence, n (\%) & 1 & $2-3$ \\
Sex & & & & \\
Men & $5736(47.3)$ & $888(15.5)$ & 14.2 & 1.3 \\
$\begin{array}{l}\text { Women } \\
\text { Annual household income (CNY) }\end{array}$ & $6391(52.7)$ & $1008(15.8)$ & 14.2 & 1.6 \\
$<10,000$ & $1374(11.3)$ & $133(9.9)$ & 9.0 & 0.9 \\
10,000 to 29,999 & $2551(21.0)$ & $352(13.8)$ & 12.7 & 1.1 \\
30,000 to 49,999 & $2086(17.2)$ & $339(16.3)$ & 14.4 & 1.9 \\
50,000 to 99,999 & $2618(21.6)$ & $418(15.9)$ & 14.6 & 1.3 \\
100,000 to 199,999 & $2466(20.3)$ & $463(18.7)$ & 16.9 & $1.8 ?$ ¿? \\
200,000 & $1032(8.5)$ & $191(18.3)$ & 16.8 & 1.5 \\
Parental highest educational level & & & & \\
Primary school and below & $846(7.0)$ & $92(11.1)$ & 10.4 & 0.7 \\
Middle school & $3128(25.8)$ & $429(13.8)$ & 12.4 & 1.4 \\
High school & $2980(24.6)$ & $482(16.1)$ & 14.4 & 1.7 \\
College and above & $5173(42.6)$ & $893(17.2)$ & 15.7 & 1.4 \\
\hline
\end{tabular}

Table 2. Association of dermographism with comorbidities and patient-reported outcomes 


\begin{tabular}{|c|c|c|c|c|c|}
\hline $\begin{array}{l}\text { Comorbidities or outcomes } \\
\text { Comorbidities }\end{array}$ & Total, n (\%) & No, $\%$ & & Adjustec \\
\hline Obesity (body mass index[?]28 kg/m²) & $572(4.8)$ & 4.8 & 4.5 & $0.93(0.74-1.18)$ & $0.91(0.7$ \\
\hline Asthma & $175(1.5)$ & 1.4 & 1.7 & $1.20(0.81-1.76)$ & $1.06(0.7$ \\
\hline Allergic rhinitis & $1324(10.9)$ & 10.8 & 11.6 & $1.08(0.92-1.26)$ & $1.00(0.8$ \\
\hline Atopic dermatitis & $422(3.5)$ & 3.3 & $4.7^{+}$ & $1.45(1.14-1.85)^{+}$ & $1.39(1.0$ \\
\hline Chronic urticaria & $247(2.0)$ & 1.6 & $4.2^{+}$ & $2.64(2.02-3.47)+$ & $2.56(1.9$ \\
\hline Chronic spontaneous urticaria & $131(1.1)$ & 0.8 & 2.6 & $3.22(2.25-4.61)^{+}$ & $3.09(2.1$ \\
\hline Chronic inducible urticaria & $116(1.0)$ & 0.8 & 1.7 & $2.02(1.34-3.05)+$ & $1.97(1$ \\
\hline Acne vulgaris & $1294(10.7)$ & 10.2 & $13.0^{+}$ & $1.31(1.13-1.51)^{+}$ & $1.29(1$ \\
\hline Vitiligo & $30(0.2)$ & 0.2 & 0.3 & $1.35(0.56-3.31)$ & $1.51(0$ \\
\hline Tinea & $437(3.6)$ & 3.5 & 3.9 & $1.11(0.86-1.43)$ & $1.13(0$ \\
\hline Warts & $62(0.5)$ & 0.4 & $0.9^{+}$ & $1.96(1.11-3.46)+$ & $2.07(1$ \\
\hline \multicolumn{6}{|l|}{ Patient-reported outcomes } \\
\hline Itch (NRS[?]3) & $1853(15.3)$ & 14.9 & $17.4^{+}$ & $1.20(1.06-1.37)^{+}$ & $1.20(1$ \\
\hline Pain (NRS[?]3) & $340(2.8)$ & 2.8 & 3.0 & $1.07(0.80-1.43)$ & $1.12(0$ \\
\hline Sleep disturbance (PSQI [?]6) & 3644 (30.1) & 29.9 & 30.9 & $1.05(0.94-1.17)$ & $1.04(0$ \\
\hline Anxiety (GAD-2 [?]3) & $1003(8.3)$ & 8.3 & 7.9 & $0.95(0.79-1.13)$ & $0.96(0$ \\
\hline Depression (PHQ-2 [?]3) & $871(7.2)$ & 7.1 & 7.7 & $1.10(0.91-1.32)$ & $1.09(0$ \\
\hline Suicidal ideation & $482(4.0)$ & 4.0 & 3.9 & $0.96(0.75-1.24)$ & $0.96(0$ \\
\hline
\end{tabular}

GAD-2, Generalized Anxiety Disorder-2; NRS: numeric rating scale; PHQ-2, 2-item Patient Health Questionnaire; PSQI, Pittsburgh Sleep Quality Index; OR, odds ratio. UI, uncertainty interval.

* Adjusted for sex, household income, and parental educational level. ${ }^{+} P<0.05$ 